\title{
REMAINDERS OF POWER SERIES
}

\author{
J.D. MCCALL \\ Department of Mathematics \\ LeMoyne-Owen College \\ Memphis, Tennessee 38126 U.S.A.
}

\section{G.H. FRICKE}

Department of Mathematics

Wright State University

Dayton, Ohio 45431 U.S.A.

\section{W.A. BEYER}

Department of Mathematics

Los Alamos Scientific Laboratory

Los Alamos, New Mexico 87545 U.S.A.

(Received February 21, 1978 and in Revised form March 20, 1979)

ABSTRACT. Suppose $\sum_{n=0}^{\infty} a_{n} z^{n}$ has radius of convergence $R$ and $\sigma_{N}(z)=$ $\left|\Sigma_{n=N}^{\infty} a_{n} z^{n}\right|$. Suppose $\left|z_{1}\right|<\left|z_{2}\right|<R$, and $T$ is either $z_{2}$ or a neighborhood of $z_{2}$. Put $S=\left\{N \mid \sigma_{N}\left(z_{1}\right)>\sigma_{N}(z)\right.$ for $\left.z \in T\right\}$. Two questions are asked: (a) can $S$ be cofinite? (b) can $\mathrm{S}$ be infinite? This paper provides some answers to these questions. The answer to (a) is no, even if $T=z_{2}$. The answer to (b) is no, for $T=z_{2}$ if $\lim a_{n}=a \neq 0$. Examples show $(b)$ is possible if $T=z_{2}$ and for $T$ a neighborhood of $z_{2}$. 
KEY WORDS AND PHRASES. Power-Series, Remainders, Radius of Convergence. AMS (MOS) SUBJECT CLASSIFICATION (1970) CODES. 30A10.

1. INTRODUCTION.

This paper originated in a question of approximation by power series raised in Query 51 in the American Mathematical Society Notices [1]. (The query originated in considerations of analytically continuing a polynomial series from the interval $[-1,1]$ to the region of convergence of the series.) Suppose $f(z)=\Sigma_{n=0}^{\infty} a_{n} z^{n}$ has radius of convergence $R$ and $\sigma_{N}(z)=\left|\Sigma_{n=N}^{\infty}{ }_{n} z^{n}\right|$. Suppose $\left|z_{1}\right|<\left|z_{2}\right|<R$ and $T$ is either $z_{2}$ or a neighborhood of $z_{2}$. Put $S=$ $\left\{n \mid \sigma_{n}\left(z_{1}\right)>\sigma_{n}(z)\right.$ for $\left.z \in T\right\} . S$ is cofinite if its complement is finite. Two questions are asked:

(a) can $S$ be cofinite?

(b) can $\mathrm{S}$ be infinite?

One might expect the answer to both questions to be no since one expects the approximation to $f$ by partial sums of its power series to be worse, closer to the circle of convergence.

This paper provides some answers to these questions. Section 2 shows (a) is impossible for any $T$. Section 3 shows (b) is impossible if $T=z_{2}$ and $\lim a_{n}=a \neq 0$. Section 4 shows (b) is possible for $T=z_{2}$ and Section 5 shows (b) is possible for $T$ a neighborhood of $z_{2}$.

Section 5 suggests the conjecture that if $T$ is a neighborhood of $z_{2}$, then $\mathrm{S}$ must be "thin." The $\mathrm{S}$ which appears in Section 5 is lacunary.

These questions can also be raised about other series of orthonormal polynomials with elliptic domains of convergence. (cf. Szegö [5], pp. 309-10). 
2. S CANNOT BE COFINITE.

The following theorem was suggested by P. Lax [3] .

THEOREM 1. If $\lim \left|a_{n}\right|^{1 / n}=1 / R<\infty, 0<\left|z_{1}\right|,\left|z_{2}\right|<R$ and $0<\delta<$ $\left|z_{2}\right| /\left|z_{1}\right|$, then the set $S=\left\{n|| \Sigma_{k=n}^{\infty} a_{k} z_{2}{ }^{k}\left|<\delta^{n}\right| \Sigma_{k=n}^{\infty} a_{k} z_{1}{ }^{k} \mid\right\}$ cannot be cofinite.

PROOF. Suppose $S$ contains a nonempty tail set $\tau$; i.e. $n \in \tau$ implies $n+1 \epsilon$ $\tau$. Then for $n \in \tau$,

$$
\begin{aligned}
\sigma_{n}\left(z_{1}\right) & \geq \sigma_{n+1}\left(z_{1}\right)-\left|a_{n}\right|\left|z_{1}\right|^{n} \geq \delta^{-(n+1)} \sigma_{n+1}\left(z_{2}\right)-\left|a_{n}\right|\left|z_{1}\right|^{n} \\
& \geq \delta^{-(n+1)}\left[\left|a_{n}\right|\left|z_{2}\right|^{n}-\sigma_{n}\left(z_{2}\right)\right]-\left|a_{n}\right|\left|z_{1}\right|^{n} \\
& \geq\left|a_{n}\right|\left[\delta^{-(n+1)}\left|z_{2}\right|^{n}-\left|z_{1}\right|^{n}\right]-\delta^{-1} \sigma_{n}\left(z_{1}\right) .
\end{aligned}
$$

Hence

$$
\left(1+\delta^{-1}\right) \sigma_{n}\left(z_{1}\right) \geq\left|a_{n}\right|\left[\delta^{-(n+1)}\left|z_{2}\right|^{n}-\left|z_{1}\right|^{n}\right] .
$$

Suppose $1 / R \neq 0$. Choose $\varepsilon>0$ so that $\left(R^{-1}+\varepsilon\right)\left|z_{1}\right|<1$ and choose $n \tau$ so large that $\left|a_{k}\right|^{1 / k}<(1 / R+\varepsilon)$ for $k \geq n$. Also choose $n$ so that $\left|a_{n}\right|^{1 / n}>$ $1 / R-\varepsilon$. Then

$$
\begin{aligned}
\frac{\left[\left(R^{-1}+\varepsilon\right)\left|z_{1}\right|\right]^{n}}{1-\left(R^{-1}+\varepsilon\right)|z|} & >\Sigma_{k=n}^{\infty}\left|a_{k}\right|\left|z_{1}\right|^{k} \geq \sigma_{n}\left(z_{1}\right) \\
& \geq \frac{\left|a_{n}\right|}{1+\delta^{-1}}\left[\delta^{-(n+1)}\left|z_{2}\right|^{n}-\left|z_{1}\right|^{n}\right]
\end{aligned}
$$




$$
\begin{aligned}
& \geq \frac{\left(R^{-1}-\varepsilon\right)^{n}}{1+\delta^{-1}}\left[\delta^{-(n+1)}\left|z_{2}\right|^{n}-\left|z_{1}\right|^{n}\right] \\
& =\frac{\left(R^{-1}-\varepsilon\right)^{n}}{1+\delta^{-1}}\left|\frac{\left|z_{2}\right|}{\delta}\right|^{n}\left[\delta^{-1}-\left(\frac{\delta\left|z_{1}\right|}{z_{2}}\right)^{n}\right] .
\end{aligned}
$$

Now in addition to the other conditions on $n$, choose $n$ large enough so that

$$
\left(\frac{\delta\left|z_{1}\right|}{\left|z_{2}\right|}\right)^{n}<\delta^{-1}
$$

Then, since

$$
\frac{\left(\mathrm{R}^{-1}+\varepsilon\right)\left|\mathrm{z}_{1}\right|}{\left[1-\left(\mathrm{R}^{-1}+\varepsilon\right)\left|\mathrm{z}_{1}\right|\right]^{1 / \mathrm{n}}} \geq \frac{\mathrm{R}^{-1}-\varepsilon}{\left(1+\delta^{-1}\right)^{1 / n}} \frac{\left|\mathrm{z}_{2}\right|}{\delta}\left[\delta^{-1}-\left(\frac{\delta\left|\mathrm{z}_{1}\right|}{\left|\mathrm{z}_{2}\right|}\right)^{\mathrm{n}}\right]^{1 / \mathrm{n}}
$$

one obtains upon letting $\varepsilon \rightarrow 0$ and $n \rightarrow \infty$ :

$$
\left|z_{1}\right| \geq \frac{\left|z_{2}\right|}{\delta}
$$

contradicting $\delta<\left|z_{2}\right| /\left|z_{1}\right|$.

Suppose $R^{-1}=0$. Then $\left|a_{n}\right|^{1 / n}$ converges to zero. If we add zero to the set, $\left\{\left|a_{n}\right|^{1 / n} \mid n \geq 1\right\}$ the new set is closed and bounded and thus compact with the largest element $\left.\left.\right|^{a} n_{1}\right|^{1 / n_{1}}$. Deleting $\left|a_{1}\right|,\left|a_{2}\right|^{1 / 2}, \ldots,\left.\left.\right|^{a} n_{1}\right|^{1 / n_{1}}$, there is a largest element $\left.\left.\right|^{a} n_{2}\right|^{1 / n_{2}}$ in the remaining set and so forth. Thus we obtain a sequence $n_{i}, i=1,2, \ldots$, with $\left|{ }^{a} n_{i}\right|^{1 / n_{i}}=\varepsilon_{i} \neq 0$ and $\left|a_{n}\right|^{1 / n} \leq \varepsilon_{i}$ for $n \geq n_{i}$. Also $\lim _{i \rightarrow \infty} \varepsilon_{i}=0$. Thus for $i$ large enough that $\varepsilon_{i}\left|z_{1}\right|<1$ : 


$$
\begin{aligned}
\frac{\left[\varepsilon_{i}\left|z_{1}\right|\right]_{i}^{n_{i}}}{1-\varepsilon_{i}\left|z_{1}\right|} & \geq \Sigma_{k=n_{i}}^{\infty}\left|a_{k}\right|\left|z_{1}\right|^{k} \geq \sigma_{n_{i}}\left(z_{1}\right) \\
& \geq \frac{\left|{ }^{a} n_{i}\right|}{1+\delta^{-1}}\left[\delta_{i}^{-\left(n_{i}+1\right)}\left|z_{2}\right|^{n_{i}}-\left|z_{1}\right|^{n_{i}}\right] \\
& =\frac{\left|n_{i}\right|}{1+\delta^{-1}} \frac{\left|z_{2}\right|}{\delta} n_{i}\left[\delta^{-1}-\left(\frac{\delta\left|z_{1}\right|}{\left|z_{2}\right|}\right)^{n_{i}}\right] .
\end{aligned}
$$

Now choose $n_{i}$ so that $\left(\delta\left|z_{1}\right| /\left|z_{2}\right|\right)^{n_{i}}<\delta^{-1}$. Then

$$
\frac{\varepsilon_{i}\left|z_{1}\right|}{\left(1-\varepsilon_{i}\left|z_{1}\right|\right)^{1 / n_{i}}} \geq \frac{\left.\right|^{a_{n} n^{1 / n_{i}}}}{\left(1+\delta^{-1}\right)^{1 / n_{i}}} \frac{\left|z_{2}\right|}{\delta}\left[\delta^{-1}-\left(\frac{\delta\left|z_{1}\right|}{\left|z_{2}\right|}\right)^{n_{i}}\right]^{1 / n_{i}}
$$

or

$$
\frac{\left|z_{1}\right|}{\left(1-\varepsilon_{i}\left|z_{1}\right|\right)^{1 / n_{i}}} \geq \frac{\left|z_{2}\right|}{\delta\left(1+\delta^{-1}\right)^{1 / n_{i}}}\left[\delta^{-1}-\left(\frac{\delta\left|z_{1}\right|}{\left|z_{2}\right|}\right)^{n_{i}}\right]^{1 / n_{i}} .
$$

Letting $\varepsilon_{i} \rightarrow 0$ and $n_{i} \rightarrow \infty$, one obtains

$$
\left|z_{1}\right| \geq \frac{\left|z_{2}\right|}{\delta}
$$

contradicting $\delta<\left|z_{2}\right| /\left|z_{1}\right|$. This completes the proof of Theorem 1 .

The following observation about general series was made by a referee. Let $\Sigma_{0}^{\infty} A_{\mu}$ be convergent. If $\Sigma_{0}^{\infty} \mu\left|b_{\mu}\right|<\infty$, then

$$
S=\left\{N|| \sum_{\mu \geq N} A_{\mu}|<| \sum_{\mu \geq N} A_{\mu} b_{\mu} \mid\right\}
$$


is not cofinite. For let $R_{n}=\Sigma_{\mu \geq n} A_{\mu}$. Then $A_{\mu}=R_{n}-R_{n+1}$. If $S$ were cofinite, then for $\mathrm{n} \geq \mathrm{n}_{\mathrm{o}}$,

$$
\left|A_{\mu}\right| \leq\left|R_{n}\right|+\left|R_{n+1}\right| \leq 2 \Sigma_{\mu \geq n}\left|A_{\mu}\right|\left|b_{\mu}\right|
$$

or

$$
\mu=N{ }_{\mu \geq N \mu \geq n}^{\left|A_{\mu}\right| \leq 2}\left|A_{\mu}\right|\left|b_{\mu}\right| \leq 2 \underset{\mu \geq N}{\mu\left|A_{\mu}\right|\left|b_{\mu}\right|<\infty .}
$$

If $N_{0}$ is selected so large that $\mu\left|b_{\mu}\right|<1 / 2$, then for $N>N_{0}$,

$$
\sum_{\mu \geq N}\left|A_{\mu}\right|<2 \frac{1}{2} \sum_{\mu \geq N}\left|A_{\mu}\right|=\sum_{\mu \geq N}\left|A_{\mu}\right|,
$$

which is a contradiction. If one puts

$$
A_{\mu}=a_{\mu} z_{2}^{\mu}, b_{\mu}=\left(\frac{z_{1}}{z_{2}}\right)^{\mu}
$$

then under the hypothesis of Theorem 1, one obtains the weaker result that the set

$$
s=\left\{n|| \sum_{k=n}^{\infty} a_{k} z_{2}{ }^{k}|<| \sum_{k=n}^{\infty} a_{k} z_{1}^{k} \mid\right\}
$$

cannot be cofinite.

3. CASE OF LIM $_{\mathrm{N} \rightarrow \infty} \mathrm{A}_{\mathrm{N}}=\mathrm{A} \neq 0$.

In this section it is shown that (b) is impossible for even a single point if $\lim _{n \rightarrow \infty} a_{n}=a \neq 0$. The proof is as follows. For $\varepsilon>0, N$ large enough, and $|z|<R=1$ 


$$
\begin{aligned}
\sigma_{N}(z) & =\left|\sum_{n=N}^{\infty} a_{n} z^{n}\right|=\left|a \sum_{n=N}^{\infty} z^{n}+\sum_{n=N}^{\infty}\left(a_{n}-a\right) z^{n}\right| \\
& \leq|a| \frac{|z|^{n}}{|1-z|}+\varepsilon \frac{|z|^{n}}{1-|z|} .
\end{aligned}
$$

Also

$$
\begin{aligned}
|a| \frac{|z|^{N}}{|1-z|} & =\left|a \sum_{n=N}^{\infty} z^{n}\right|=\left|\sum_{n=N}^{\infty} a_{n} z^{n}+\sum_{n=N}^{\infty}\left(a-a_{n}\right) z^{n}\right| \\
& \leq \sigma_{n}(z)+\varepsilon \frac{|z|^{N}}{1-|z|} .
\end{aligned}
$$

Thus

$$
|a| \frac{|z|^{N}}{|1-z|}-\varepsilon \frac{|z|^{N}}{1-|z|} \leq \sigma_{N}(z) \leq|a| \frac{|z|^{N}}{|1-z|}+\varepsilon \frac{|z|^{N}}{1-|z|} .
$$

Suppose $\sigma_{N}\left(z_{2}\right)<\sigma_{N}\left(z_{1}\right)$ for infinitely many $N$. Then (1) gives

$$
\begin{aligned}
|a| \frac{\left|z_{2}\right|^{N}}{\left|1-z_{2}\right|}-\varepsilon \frac{\left|z_{2}\right|^{N}}{1-\left|z_{2}\right|} & \leq \sigma_{N}\left(z_{2}\right)<\sigma_{N}\left(z_{1}\right) \\
& \leq|a| \frac{\left|z_{1}\right|^{N}}{\left|1-z_{1}\right|}+\varepsilon \frac{\left|z_{1}\right|^{N}}{1-\left|z_{1}\right|}
\end{aligned}
$$

for infinitely many $N$. Taking Nth roots, letting $N \rightarrow \infty$, and $\varepsilon \rightarrow 0$, yields

$$
\left|z_{2}\right| \leq\left|z_{1}\right|
$$

a contradiction of $\left|z_{1}\right|<\left|z_{2}\right|$. 
4. FOR $\mathrm{T}=\left\{\mathrm{z}_{2}\right\}$, (b) IS POSSIBLE.

The following example shows (b) is possible if $T=\left\{z_{2}\right\}$. Let

$$
\begin{aligned}
F(z) & =(1-2 z)\left(1-z^{2}\right)^{-1} \\
& =1-2 z+z^{2}-2 z^{3}+z^{4}-2 z^{5}+\ldots .
\end{aligned}
$$

One has:

$$
\begin{aligned}
\sigma_{2 k}(z) & =\left|z^{2 k}-2 z^{2 k+1}+z^{2 k+2}-2 z^{2 k+3}+\ldots\right| \\
& =|z|^{2 k}\left|1-2 z+z^{2}-2 z^{3}+\ldots\right| \\
& =|z|^{2 k}|1-2 z|\left|1-z^{2}\right|^{-1}
\end{aligned}
$$

and thus $\sigma_{2 k}(1 / 2)=0$. So for any $z_{1} \neq 1 / 2$ and $0<\left|z_{1}\right|<1, \sigma_{2 k}\left(z_{1}\right)>$ $\sigma_{2 \mathrm{k}}(1 / 2)$.

Note that for an $\varepsilon$-neighborhood of $1 / 2: \quad N=\{z|| z-1 / 2 \mid<\varepsilon\}$, $0<\varepsilon<1 / 2$ and for any $z_{1}$ with $\left|z_{1}\right|<1 / 2-\varepsilon, \sigma_{2 k}\left(z_{1}\right)$ converges to zero faster than $\sigma_{2 k}(z)$ at any point $z$ in $N$ except $1 / 2$. So we cannot extend the result to a neighborhood of $1 / 2$.

5. CASE OF $T$ A NEIGHBORHOOD OF $z_{2}$.

THEOREM 2. For each $R, 0<R \leq \infty$, there exist points $z_{1}$ and $z_{2}$ with $\left|z_{1}\right|<\left|z_{2}\right|<R$ and a power series $\sum_{n=0}^{\infty} a_{n} z^{n}$ with radius of convergence $R$ such that for infinitely many values of $N, \sigma_{N}\left(z_{1}\right) / 3 \geq \sigma_{N}(z)$ for all $z$ in some neighborhood of $\mathrm{z}_{2}$.

PROOF. Suppose $R=1$. Put $n_{k}=4^{k}$ and $P_{k}(z)=\left(1 / b_{k}\right) z^{n} 2 k-1(z-1 / 2)^{n} 2 k$, where $b_{k}=\max \underset{0 \leq j \leq n}{ }\left\{k\left\{\begin{array}{c:c}n_{2 k} & 2^{-j} \\ j\end{array}\right.\right.$, . The power series $\Sigma_{k=1}^{\infty} P_{k}(z)=\Sigma_{n=0}^{\infty}{ }^{a} z^{n}$ will be shown to satisfy the Theorem for $R=1$ with $z_{1}=-1 / 4$ and $z_{2}=1 / 2$. Note that 


$$
\mathrm{n}_{2 \mathrm{k}}+\mathrm{n}_{2 \mathrm{k}-1}<\mathrm{n}_{2 \mathrm{k}+1}
$$

and

$$
n_{2 k-1}(\log 4 / \log 3+1)<n_{2 k}
$$

for all k. (2) implies that each $a_{n}$ is either zero or appears exactly once as a coefficient in the expansion of some $P_{k}(z)$. Let $j_{k}$ be the integer for which $\max _{0 \leq j \leq n_{2 k}}\left\{\left(\begin{array}{c}n_{2 k} \\ j\end{array}\right) \quad 2^{-j}\right\}$ is obtained. Then

$$
\left|a_{j+n_{2 k-1}}\right|^{1 /\left(j+n_{2 k-1}\right)}=\left(\frac{\left(\begin{array}{c}
n_{2 k} \\
j
\end{array}\right) 2^{-j}}{\left(\begin{array}{c}
n_{2 k} \\
j_{k}
\end{array}\right)^{-j} k}\right)^{1 /\left(j+n_{2 k-1}\right)} \cdot\left(0 \leq j \leq n_{2 k}\right)
$$

This is less than or equal to one for all $j$ and equal to one for $j=j_{k}$, which implies the radius of convergence is one.

For all z with $|z-1 / 2|<1 / 4$ :

$$
\begin{aligned}
\left|P_{k+1}(z)\right| & =\frac{1}{b_{k+1}}|z|^{n_{2 k+1}}|z-1 / 2|^{n_{2 k+2}} \\
& <\frac{1}{b_{k}}|z|^{n_{2 k-1}}|z-1 / 2|^{n_{2 k}}|z-1 / 2|^{n_{2 k+2}-n_{2 k}} \\
& \leq\left|P_{k}(z)\right|(1 / 4)^{n_{2 k+2}-n_{2 k}} \\
& \leq(1 / 4)\left|P_{k}(z)\right| .
\end{aligned}
$$

Next, for $|z-1 / 2|<1 / 4$,

$$
\frac{\left|P_{k}(z)\right|}{\left|P_{k}(-1 / 4)\right|}=|z|^{n_{2 k-1}}|z-1 / 2|^{n_{2 k}} 4^{n_{2 k-1}}(4 / 3)^{n_{2 k}}
$$




$$
<4^{-n_{2 k}} 4^{n_{2 k-1}}(4 / 3)^{n_{2 k}}
$$

$$
=4^{n_{2 k-1}} 3^{-n_{2 k}}<1 / 4
$$

by (3). Hence, for $\mid z-1 / 2)<1 / 4$,

$$
\begin{aligned}
\sigma_{n_{2 k-1}}(z) & =\left|\sum_{j=n_{2 k-1}}^{\infty} a_{j} z^{j}\right| \leq \sum_{j=k}^{\infty}\left|P_{j}(z)\right| \\
& \leq\left|\sum_{j=k}^{\infty} 4^{k-j}\right|\left|P_{k}(z)\right| \text { by }(4) \\
& =(4 / 3)\left|P_{k}(z)\right|<(1 / 3)\left|P_{k}(-1 / 4)\right| \text { by } \quad(5) \\
& \leq(1 / 3)\left|\sum_{j=k}^{\infty} b_{j}^{-1}(-1 / 4) n_{2 j-1}(-3 / 4)^{2 j}\right| \\
& =(1 / 3) \sigma_{n_{2 k-1}}(-1 / 4),
\end{aligned}
$$

since all $n_{j}$ 's are even. This shows that the assertion holds for $z_{1}=-1 / 4$ and $z_{2}=1 / 2$.

For the case $0<R<\infty$, use the power series $\sum_{n=0}^{\infty} a_{n}(z / R)^{n}$. Then the result holds for $z_{1}=-R / 2, z_{2}=R / 2$, and the neighborhood $|z-R / 2|<R / 4$.

For the case $R=\infty$, let

$$
b_{k}=\left(n_{2 k-1}\right)^{n_{2 k-1}}{\underset{j}{j_{k}}}^{n_{2 k}} 2^{-j_{k}} .
$$

For $0 \leq \mathrm{j} \leq \mathrm{n}_{2 \mathrm{k}}$ : 


$$
\begin{aligned}
\left.\left.\right|_{j+n_{2 k-1}}\right|^{1 /\left(j+n_{2 k-1}\right)} & =\left(\frac{\sum_{2 k}^{j} 2^{-j}}{\left.\left(n_{2 k-1}\right)^{n_{2 k-1}\left(\begin{array}{l}
n_{2 k} \\
j_{k}
\end{array}\right)^{-j_{k}}}\right)^{1 /\left(j+n_{2 k-1}\right)}}\right. \\
& \leq\left(n_{2 k-1}\right)^{-n_{2 k-1} /\left(j+n_{2 k-1}\right)} \\
& \leq\left(n_{2 k}\right)^{-n_{2 k-1} /\left(n_{2 k}+n_{2 k-1}\right)} \\
& =\left(n_{2 k}\right)^{-1 / 5} \rightarrow 0 .
\end{aligned}
$$

as $k \rightarrow \infty$ and hence $\overline{\lim }\left|a_{n}\right|^{1 / n}=0$. The rest of the proof follows the case $\mathrm{R}=1$.

\section{AVERAGE REMAINDER}

Suppose $\Sigma a_{n} z^{n}$ has a radius of convergence $R$. It follows from results in Pólya and Szegö [4, Part III, problems 307-310] that the geometric mean:

$$
G^{N}(r)=\exp \left(\frac{1}{2 \pi} \int_{0}^{2 \pi} \log \sigma_{N}\left(r e^{i \theta}\right) d \theta\right),(r<R)
$$

and the pth mean, $\mathrm{p}>0$ :

$$
I_{p}^{N}(r)=\frac{1}{2 \pi} \int_{0}^{2 \pi} \sigma_{N}^{p}\left(r e^{i \theta}\right) d \theta,(r<R)
$$

are both monotone increasing functions of $r$ for each $N$ and $\log G^{N}(r)$ and $\log$ $I_{p}^{N}(r)$ are convex functions of $\log r$. Thus in the geometric mean sense and pth mean sense, $\sigma_{\mathrm{N}}(\mathrm{z})$ become larger as one approaches the circle of convergence. 


\section{ACKNOWLEDGMENT}

The authors thank Dr. Leon Heller of the Los Alamos Scientific Laboratory, who proposed this problem, for many discussions and contributions.

\section{REFERENCES}

1. Beyer, W. A. and L. Heller, Query 51, Amer. Math. Soc. Notices 21 (1974), 280 .

2. Fricke, G. H., Answer to Query 51, Amer. Math. Soc. Notices 22 (1975), 72.

3. Lax, P., private communication, June 14, 1974.

4. Pólya, G. and G. Szegö, Problems and Theorems in Analysis, Vol. 1, Springer-Verlag, New York, 1972.

5. Szegö, G., Orthogonal Polynomials, Amer. Math. Soc. Colloquium Publications 23, (1959). 


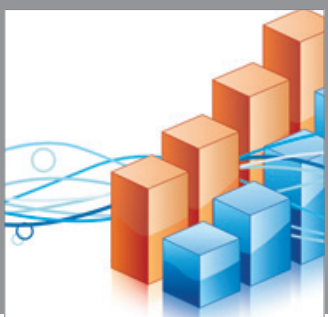

Advances in

Operations Research

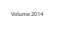

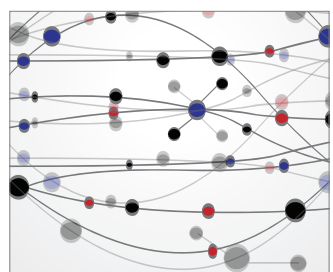

\section{The Scientific} World Journal
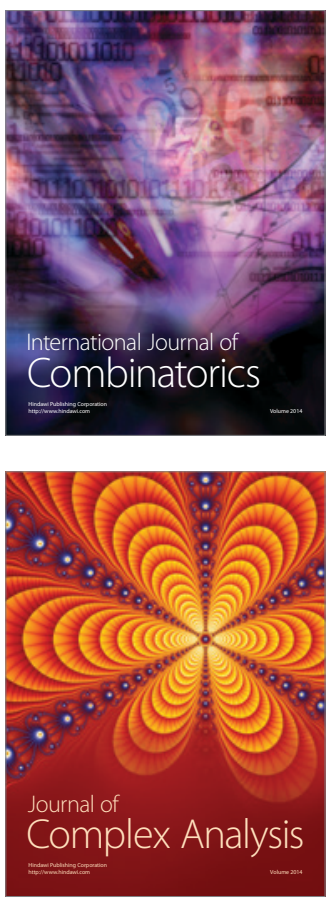

International Journal of

Mathematics and

Mathematical

Sciences
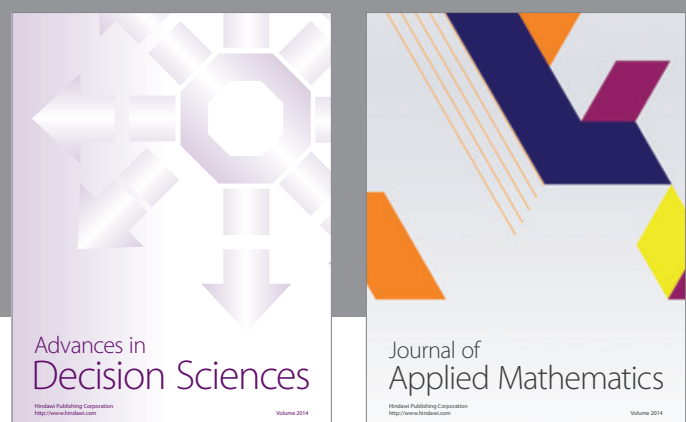

Journal of

Applied Mathematics
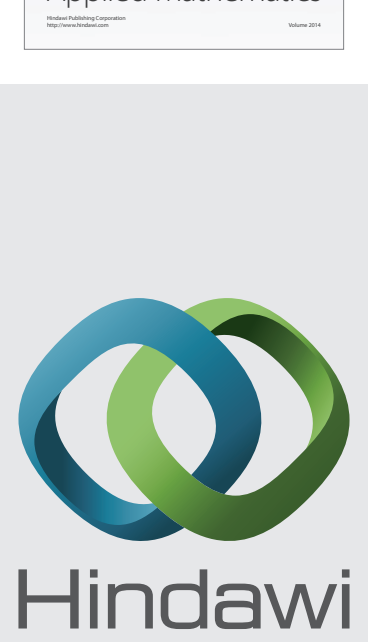

Submit your manuscripts at http://www.hindawi.com
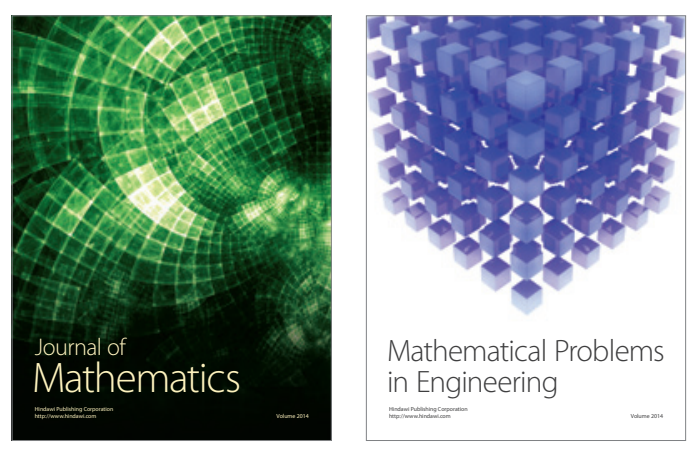

Mathematical Problems in Engineering
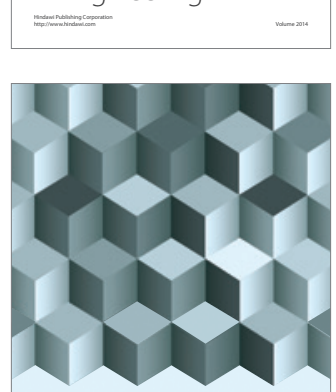

Journal of

Function Spaces
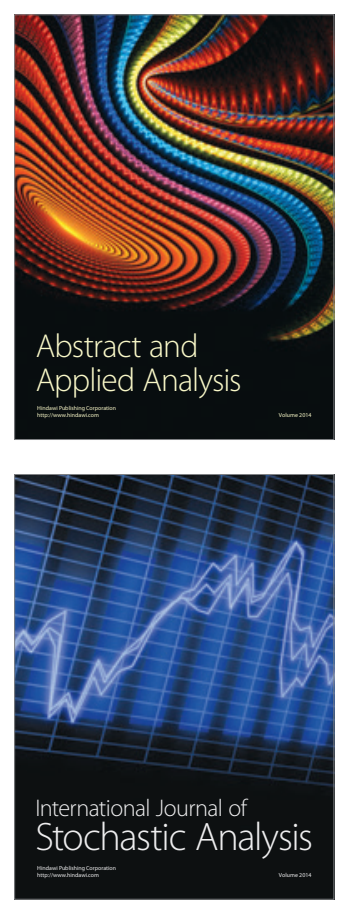

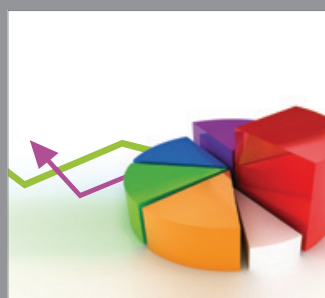

ournal of

Probability and Statistics

Promensencen
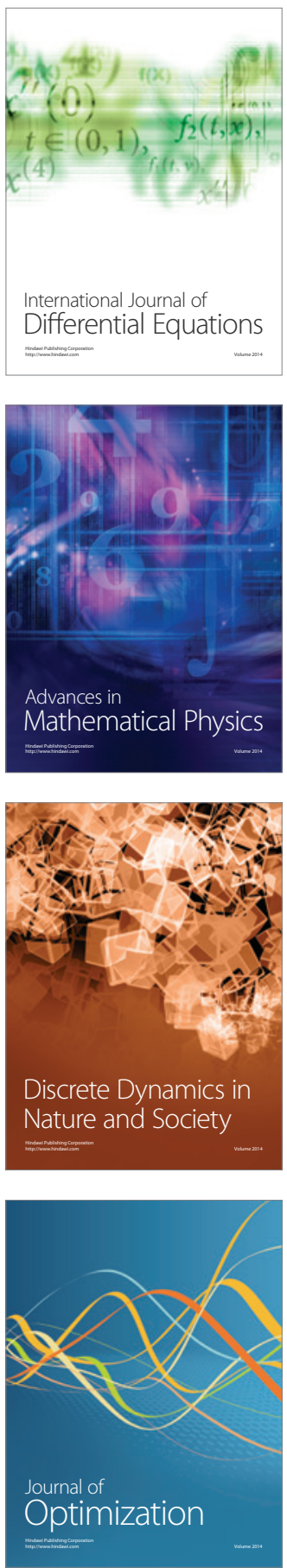